Supporting Information

\title{
Injectable Reactive Oxygen Species-Responsive SN38 Prodrug Scaffold with Checkpoint Inhibitors for Combined Chemoimmunotherapy
}

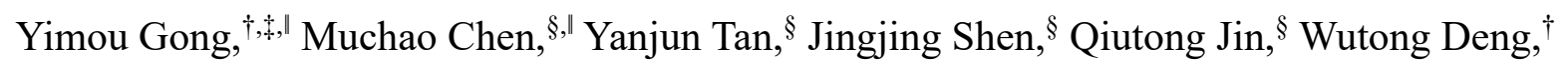
Jian Sun, ${ }^{*} \dagger,+$ Chao Wang, ${ }^{\dagger}$ Zhuang Liu $^{\S}$ and Qian Chen $^{*}, \S$

$\dagger$ Natural Products Research Center, Chengdu Institute of Biology, Chinese Academy of Sciences, Chengdu 610041, Sichuan, P. R. (China).

+ University of Chinese Academy of Sciences, Beijing 100049, P. R. (China).

$\S$ Institute of Functional Nano \& Soft Materials (FUNSOM), Jiangsu Key Laboratory for Carbon-Based Functional Materials \& Devices, Soochow University, Suzhou 215123, Jiangsu, P. R. (China).

\| These authors contributed equally.

* Correspondence to be addressed to: sunjian@cib.ac.cn, chenqian@suda.edu.cn 
Table of contents

Scheme S1. S-3

Table S1. $\quad$ S-3

$\begin{array}{ll}\text { Figure S1. } & \text { S-4 }\end{array}$

$\begin{array}{ll}\text { Figure S2. } & \text { S-5 }\end{array}$

$\begin{array}{ll}\text { Figure S3. } & \text { S-6 }\end{array}$

$\begin{array}{ll}\text { Figure S4. } & \text { S-7 }\end{array}$

$\begin{array}{lr}\text { Figure S5. } & \text { S-7 }\end{array}$

$\begin{array}{ll}\text { Figure S6. } & \text { S-8 }\end{array}$

$\begin{array}{ll}\text { Figure S7. } & \text { S-9 }\end{array}$

$\begin{array}{ll}\text { Figure S8. } & \text { S-9 }\end{array}$

$\begin{array}{lr}\text { Figure S9. } & \text { S-10 }\end{array}$

$\begin{array}{lr}\text { Figure S10. } & \text { S-10 }\end{array}$

Figure S11. $\quad$ S-11

Figure S12. $\quad$ S-11

$\begin{array}{lr}\text { Figure S13. } & \text { S-12 }\end{array}$

$\begin{array}{ll}\text { Figure S14. } & \text { S-12 }\end{array}$

$\begin{array}{lr}\text { Figure S15. } & \text { S-13 }\end{array}$

$\begin{array}{ll}\text { Figure S16. } & \text { S-13 }\end{array}$

$\begin{array}{lr}\text { Figure S17. } & \text { S-14 }\end{array}$

Figure S18 .S-14 
Scheme S1. Synthesis route of SN38-SA-BA.
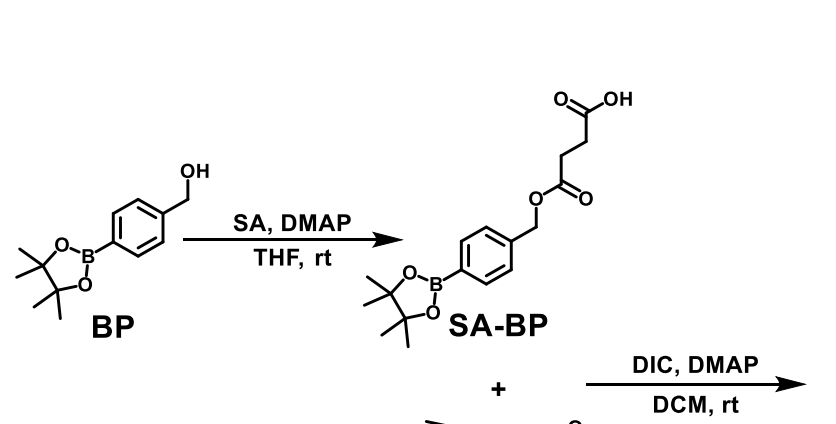<smiles>C/C=C\C1OC(C)(C)C(C)(C)O1</smiles>

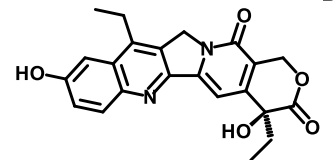

SN38<smiles>[C+]1C=CC2=CC1C2</smiles>

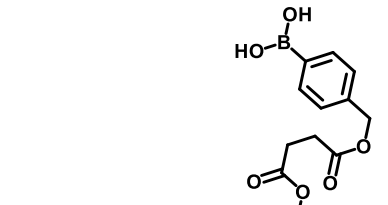
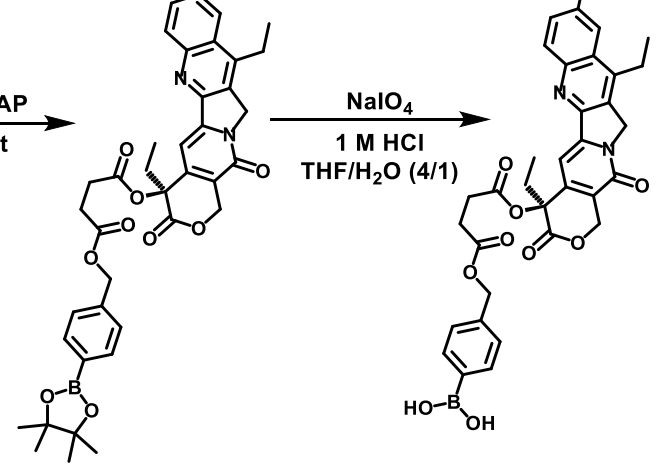

SN38-SA-BP

SN38-SA-BA

Table S1. Gelation of different proportions among PVA, SN38-SA-BA and PEG800.

\begin{tabular}{ccccc}
\hline \multirow{2}{*}{ Entry } & \multicolumn{2}{c}{ Part I } & \multicolumn{2}{c}{ Part II } \\
\cline { 2 - 4 } & $\mathrm{A}(\mu \mathrm{L})$ & $\mathrm{H}_{2} \mathrm{O}(\mu \mathrm{L})$ & $\mathrm{B}(\mu \mathrm{L})$ & PEG800 $(\mu \mathrm{L})$ \\
\hline 1 & & 50 & 30 \\
2 & & & 40 & 40 \\
3 & \multirow{2}{*}{70} & 30 & 50 \\
4 & & & 25 & 55 \\
5 & & 12.5 & 67.5 \\
6 & & 0 & 80 \\
\hline 7 & & 50 & 40 \\
8 & & & 40 & 50 \\
9 & \multirow{2}{*}{60} & 30 & 60 \\
10 & & 25 & 65 \\
11 & & & 12.5 & 77.5 \\
12 & & & 0 & 90 \\
\hline 13 & & 50 & 50 \\
14 & & & 40 & 60 \\
15 & \multirow{2}{*}{150} & 50 & 30 & 70 \\
16 & & & 25 & 75 \\
17 & & & 12.5 & 87.5 \\
18 & & & 0 & 100 \\
\hline
\end{tabular}




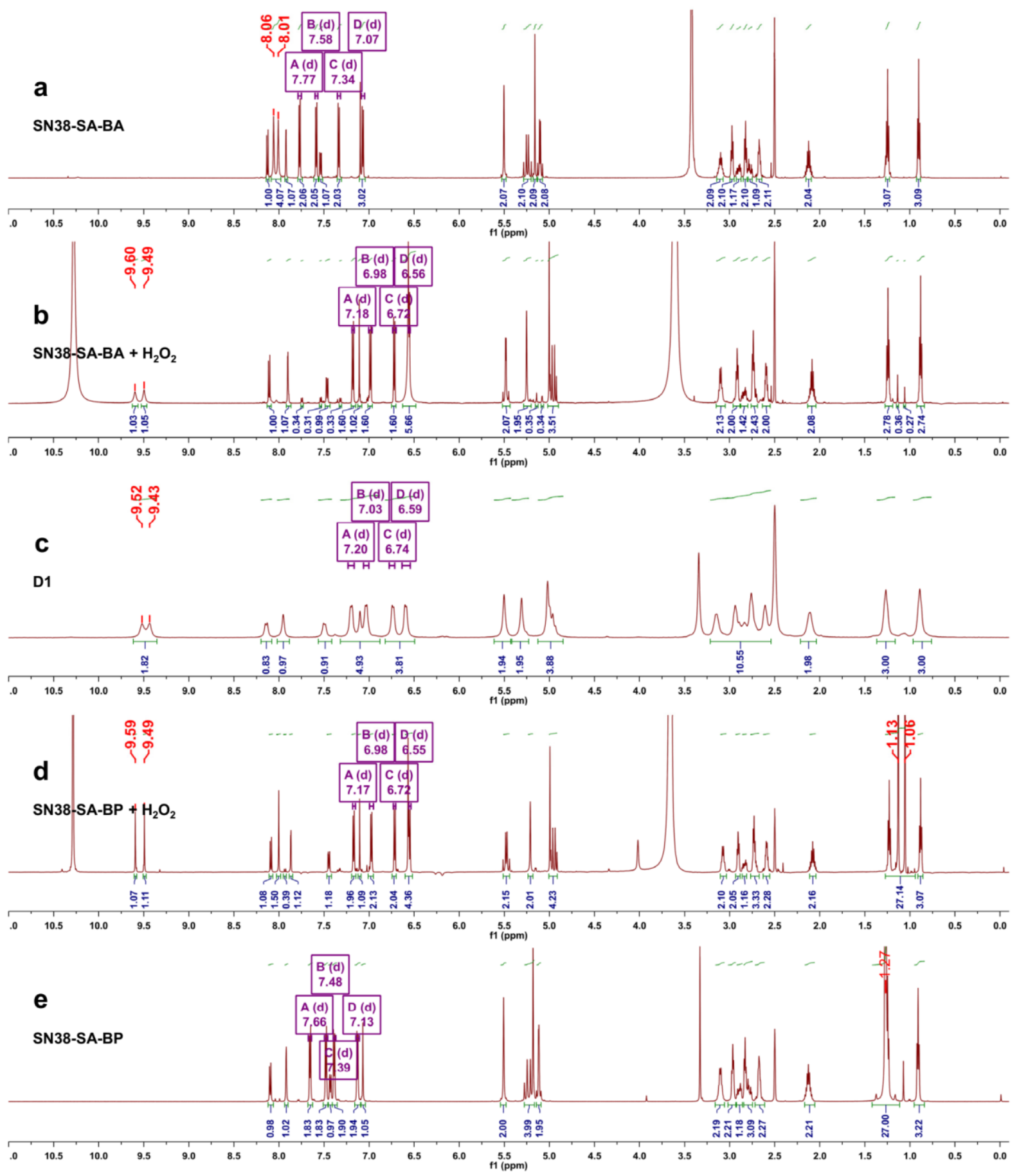

Figure S1. In situ ${ }^{1} \mathrm{H}$ NMR spectra of SN38-SA-BA (BP) dispersed in solution containing $\mathrm{H}_{2} \mathrm{O}_{2}$. DMSO-d6: (a) SN38-SA-BA. (b) SN38-SA-BA with 10-folds excess $\mathrm{H}_{2} \mathrm{O}_{2}$. (c) Intermediate D1. (d) SN38-SA-BP with 10-folds excess $\mathrm{H}_{2} \mathrm{O}_{2}$. (e) SN38-SA-BP. 


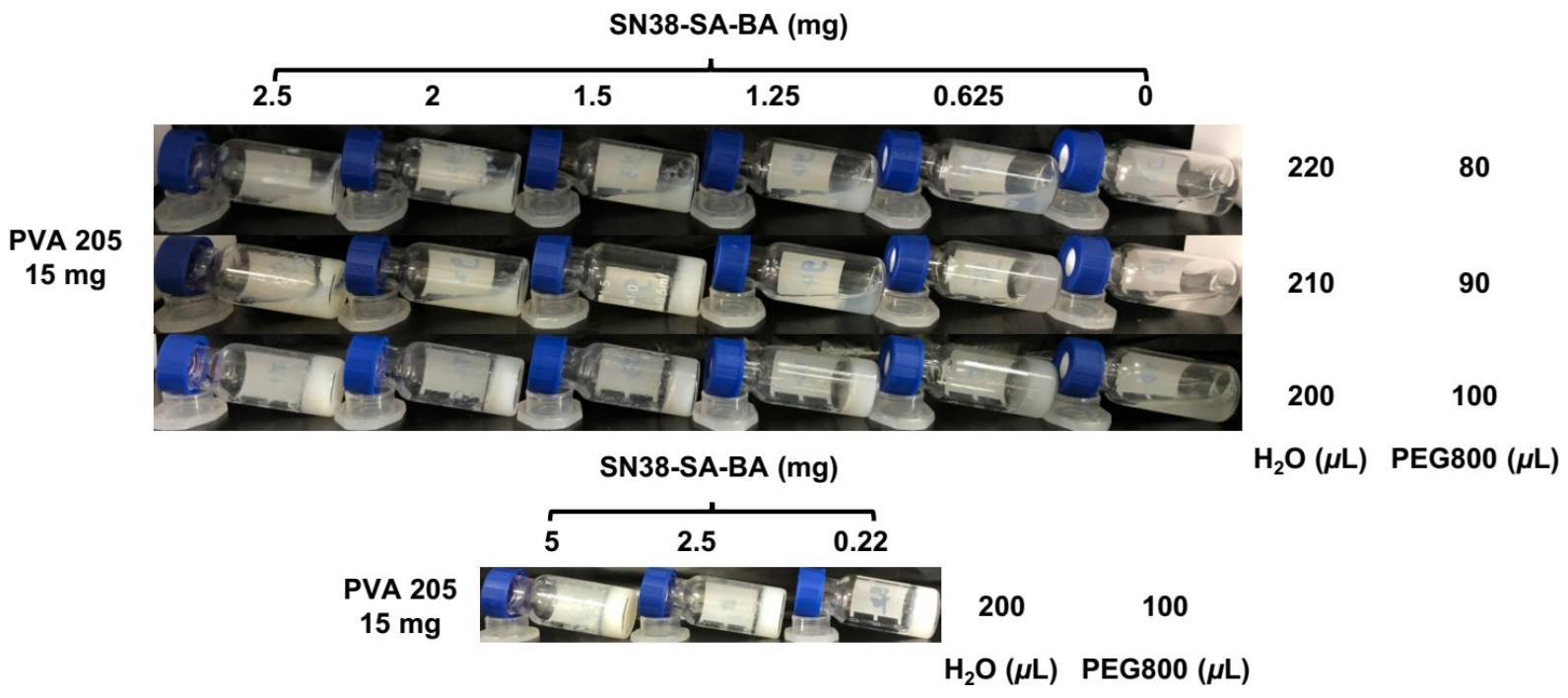

Figure S2. Fabrication of PVA-SN38 hydrogel. Photographs showing the gels of different proportion among PVA, SN38-SA-BA and PEG 800. 
a
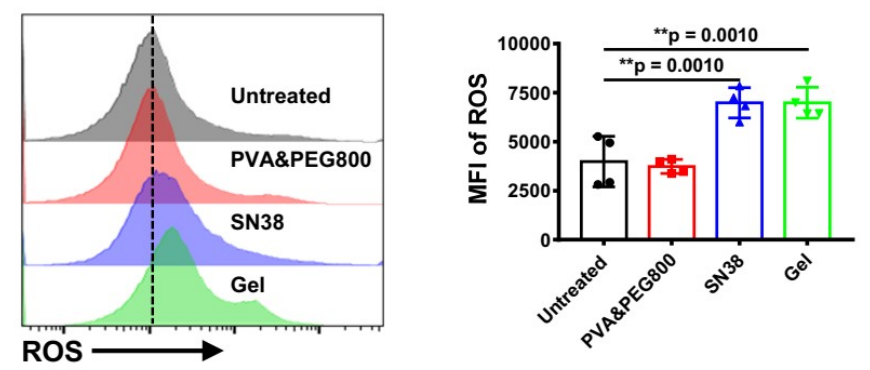

b

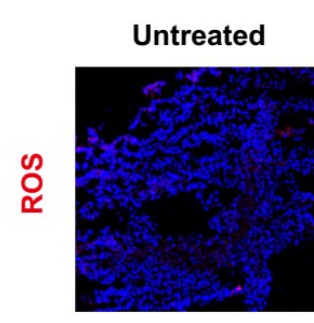

PVA\&PEG800

SN38
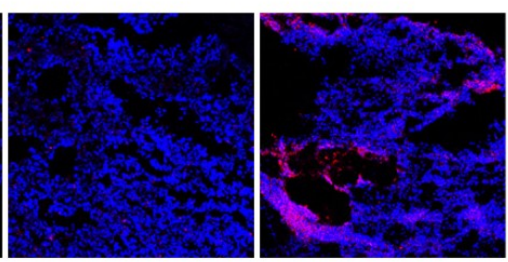

Gel
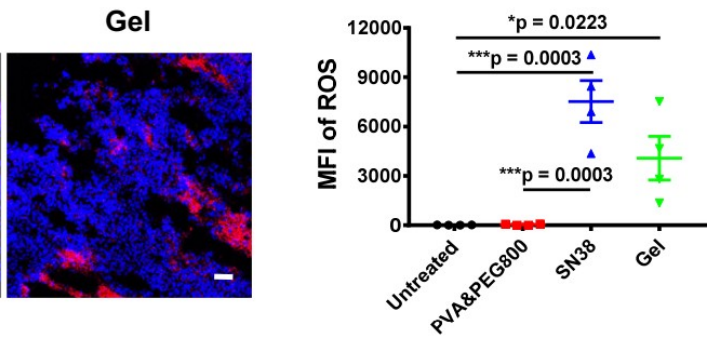

C

PVA\&PEG800

SN38

Gel
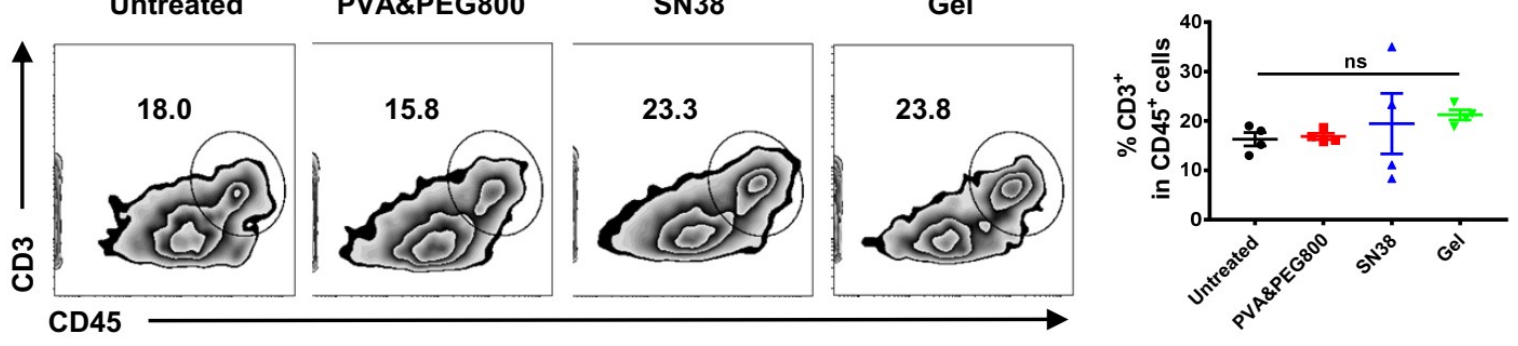

d
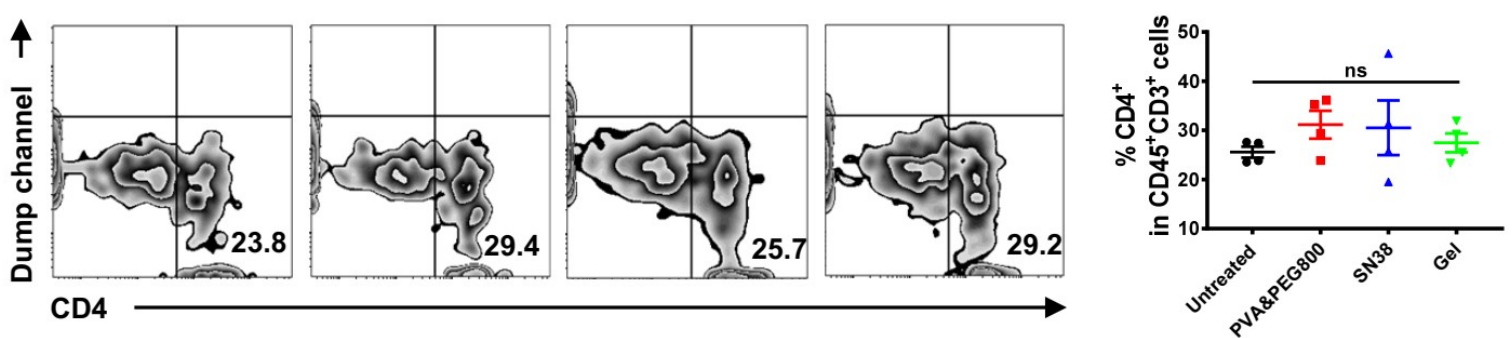

Figure S3. Immune responses induced by PVA-SN38 hydrogel in vivo. (a) Determination of intratumor total ROS level based on FACS. (b) Representative CLSM images (left) and relative semi-quantification (right) of ROS in the tumor after different treatments. DAPI, blue color. Scale bar, $50 \mu \mathrm{m}$. (c, d) Representative FACS plots (left) and the corresponding percentage (right) of $\mathrm{CD}^{+} \mathrm{T}$ cells (c), gating on $\mathrm{CD}^{2} 5^{+}$cells. And $\mathrm{CD} 4^{+} \mathrm{T}$ cells (d), gating on $\mathrm{CD}^{+} 5^{+} \mathrm{CD}^{+}$ $\mathrm{T}$ cells. Data are shown as mean $\pm \operatorname{SEM}(\mathrm{n}=4)$. Statistical significance was calculated by oneway ANOVA with Tukey's post test. ${ }^{*} P<0.05 ;{ }^{* *} P<0.01 ;{ }^{* * *} P<0.001$; ns, not significant. 
a

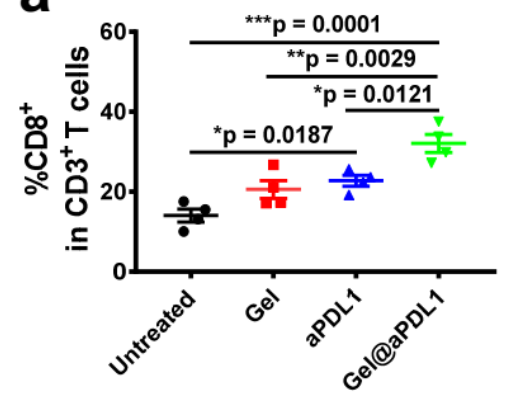

b

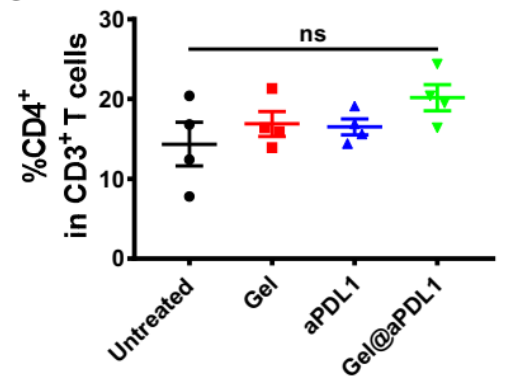

Figure S4. Percentage of CD $8^{+} \mathrm{T}$ cells (a) and $\mathrm{CD} 4^{+} \mathrm{T}$ cells $(\mathrm{b})$ in the $\mathrm{CD} 3^{+} \mathrm{T}$ cells of the tumor after various treatments in vivo. Data are shown as mean $\pm \operatorname{SEM}(n=4)$. Statistical significance was calculated by one-way ANOVA with Tukey's post test. ${ }^{*} P<0.05 ;{ }^{*} P<0.01$; ${ }^{* * *} P<$ 0.001 ; ns, not significant.

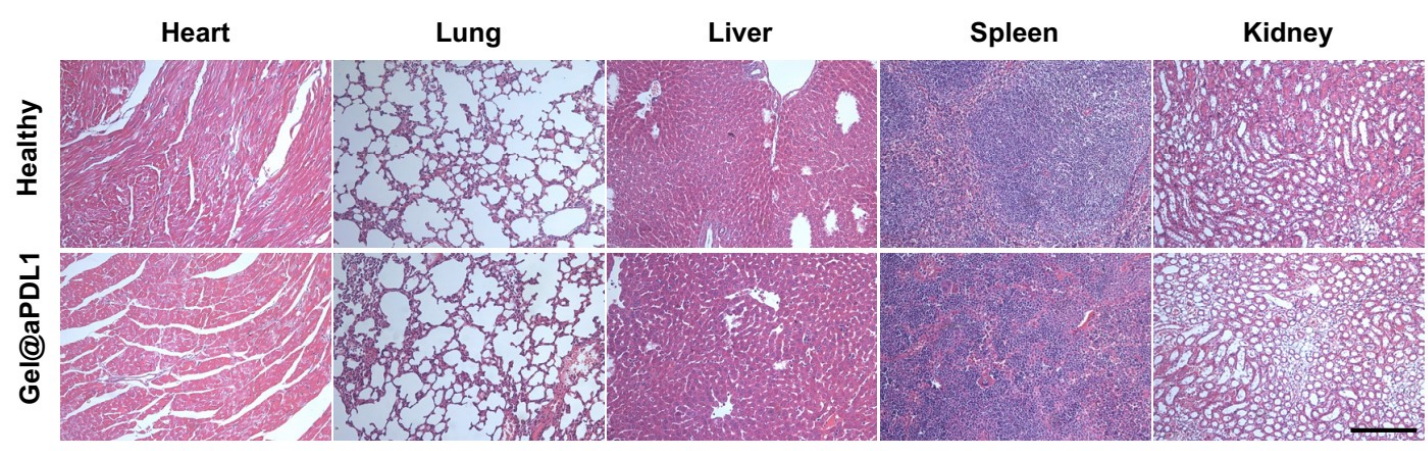

Figure S5. Biocompatibility of PVA-SN38 hydrogel in vivo. H\&E stained micrographs of major organs collected from healthy and Gel@aPDL1 treated mice on day 30. Scale bar, 200 $\mu \mathrm{m}$. 


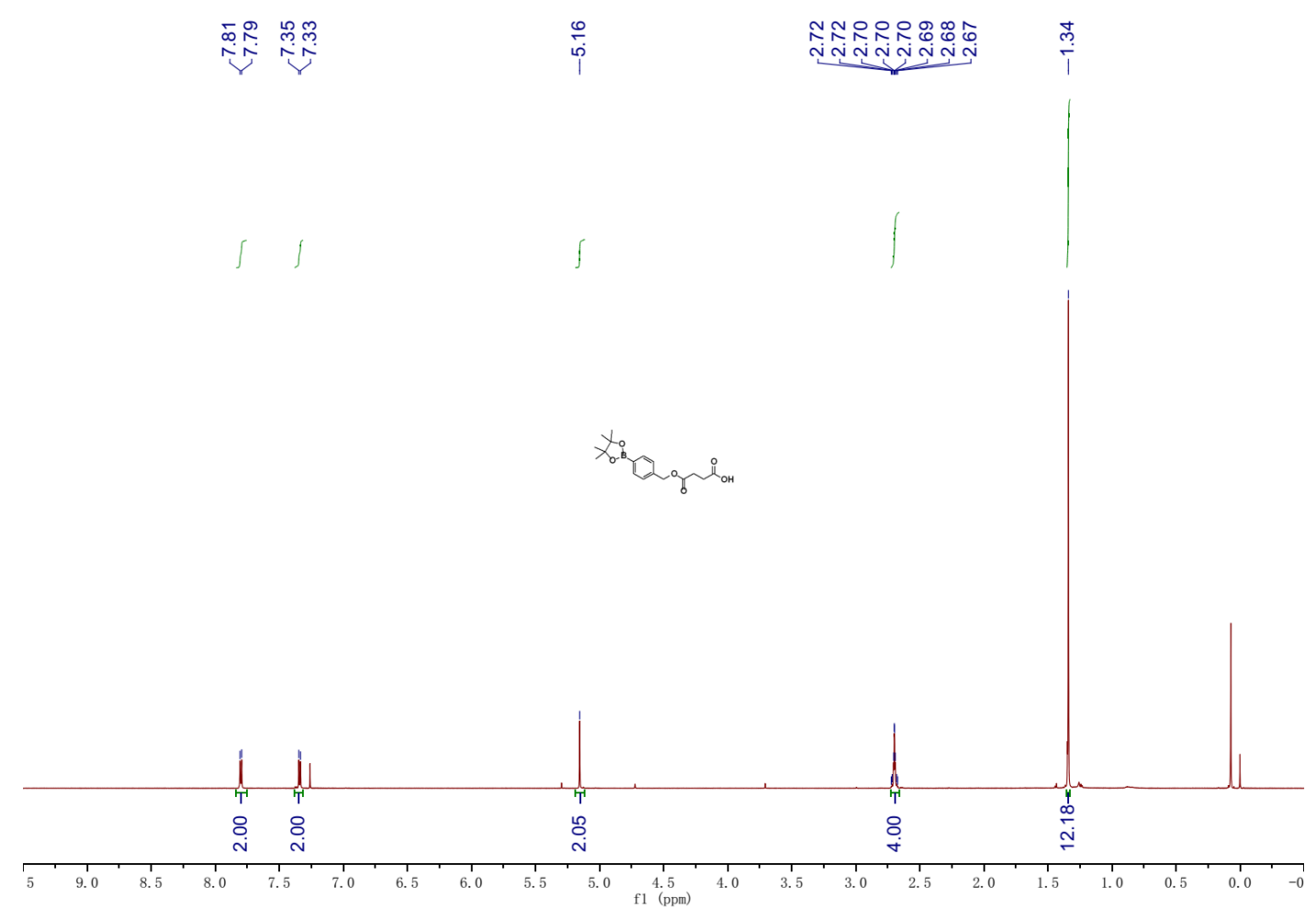

Figure S6. ${ }^{1} \mathrm{H}$ NMR spectrum of SA-BP $\left(\mathrm{CDCl}_{3}\right)$. 


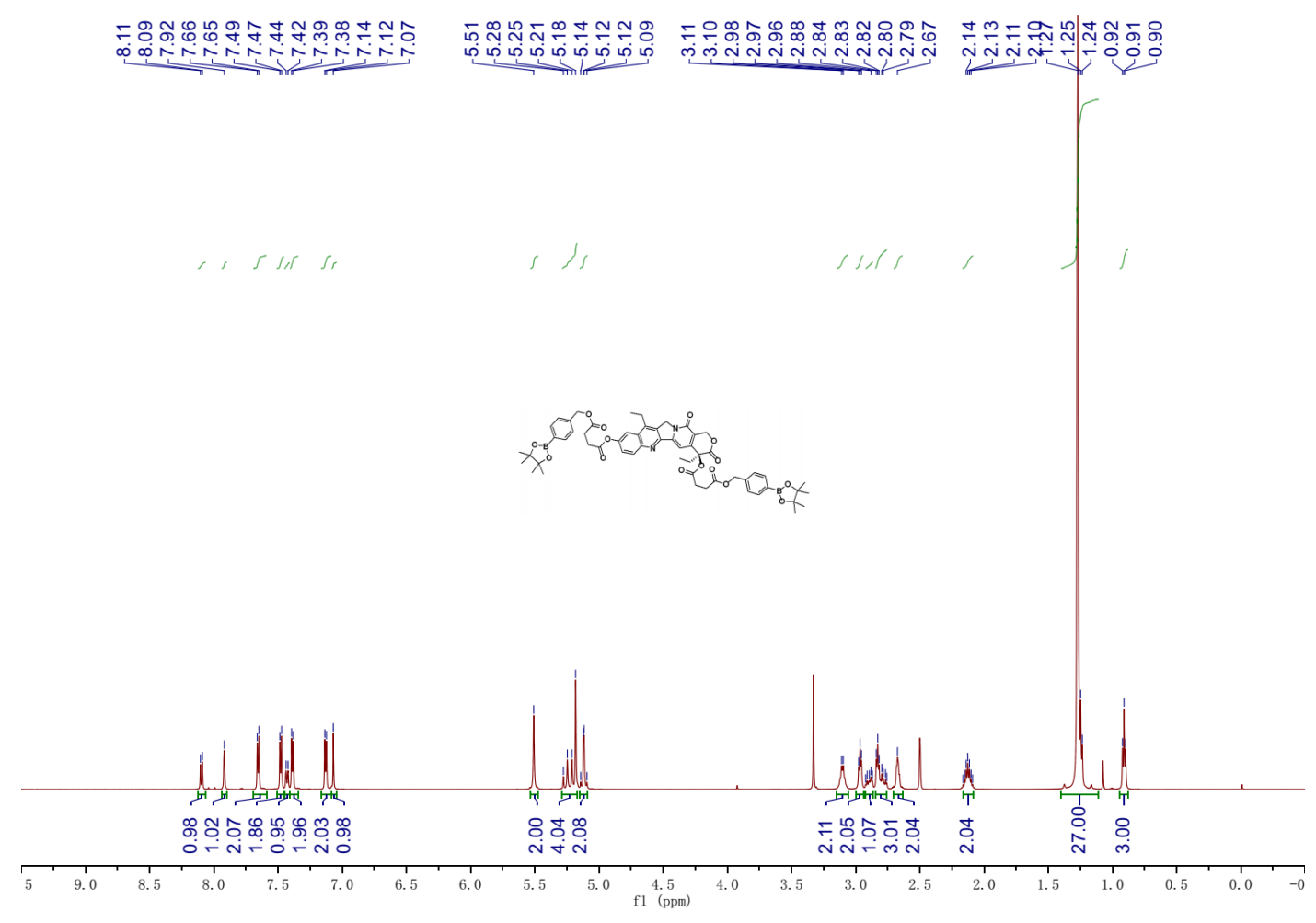

Figure S7. ${ }^{1} \mathrm{H}$ NMR spectrum of SN38-SA-BP (DMSO-d6).

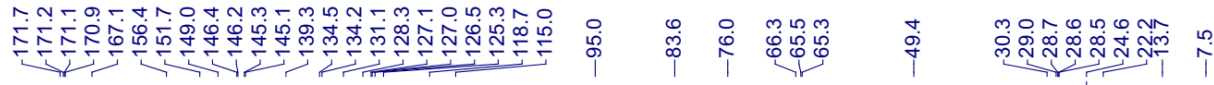

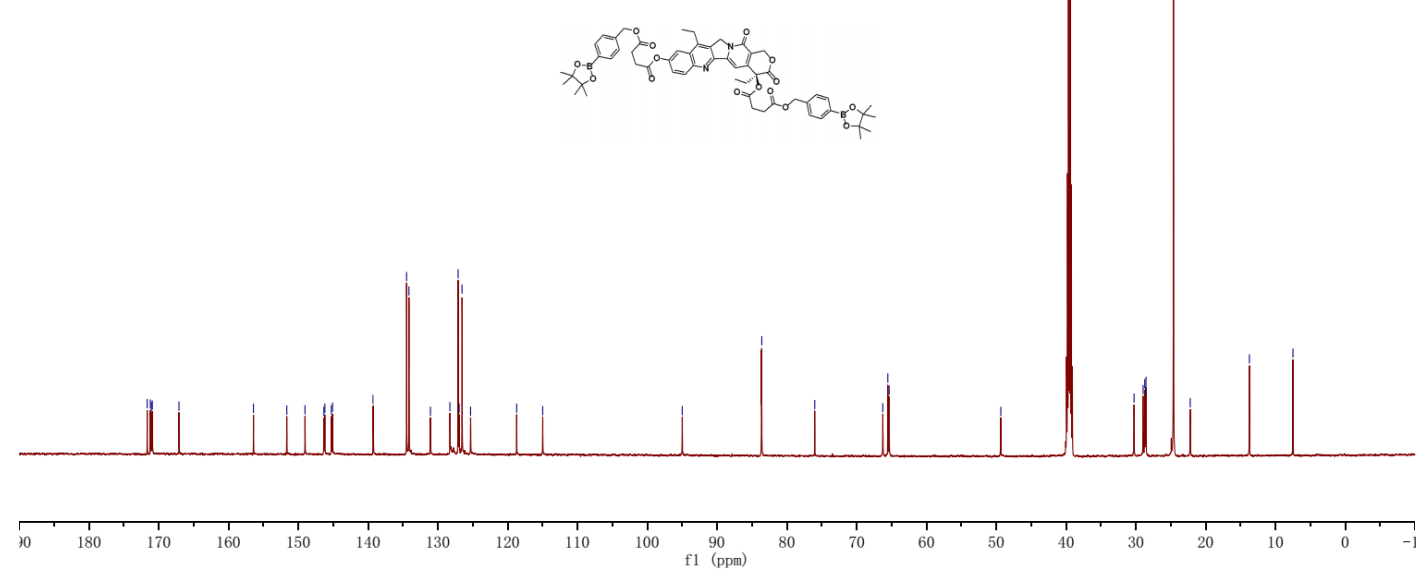

Figure S8. ${ }^{13} \mathrm{C}$ NMR spectrum of SN38-SA-BP (DMSO-d6). 


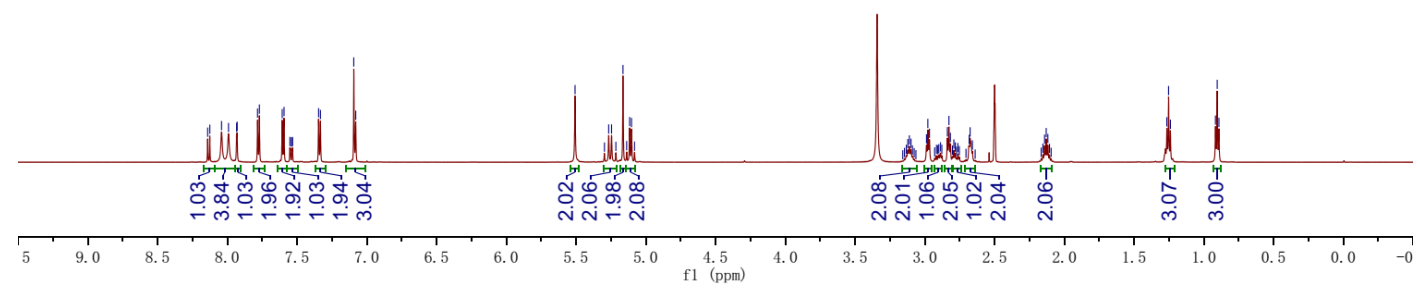

Figure S9. ${ }^{1} \mathrm{H}$ NMR spectrum of SN38-SA-BA (DMSO-d6).
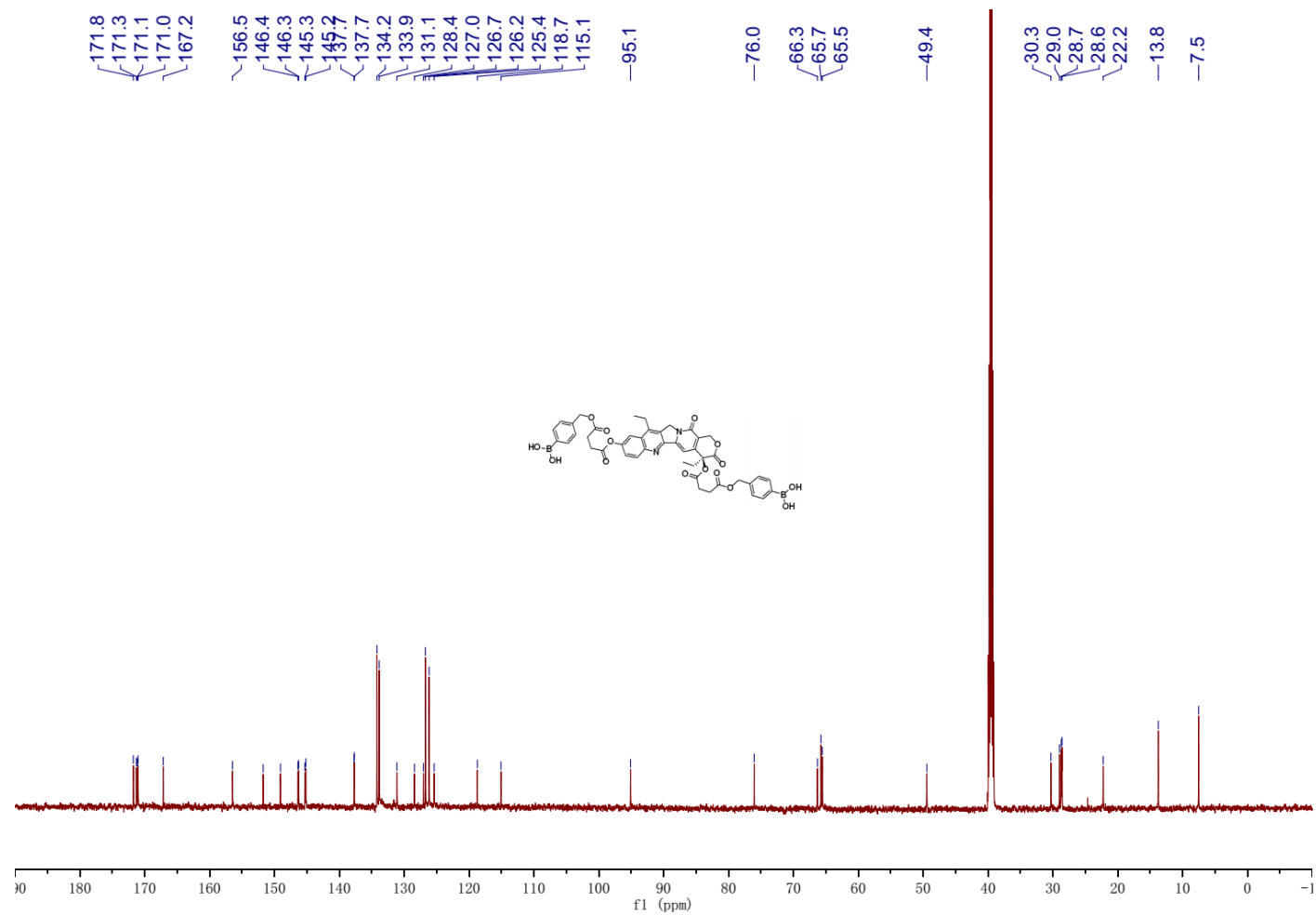

Figure S10. ${ }^{13} \mathrm{C}$ NMR spectrum of SN38-SA-BA (DMSO-d6). 

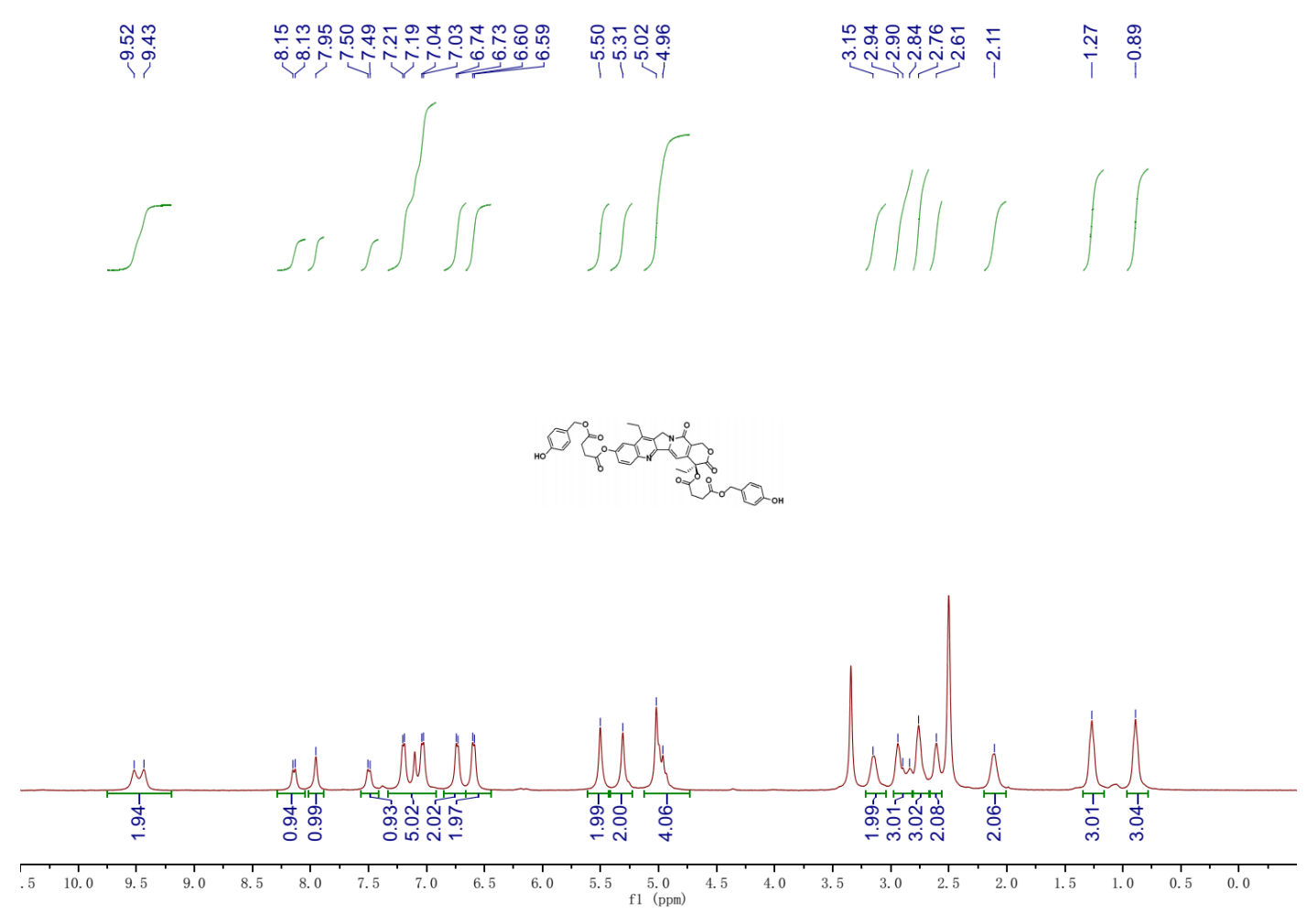

Figure S11. ${ }^{1}$ H NMR spectrum of D1 (DMSO-d6).

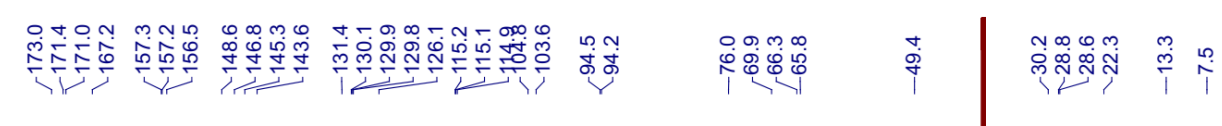

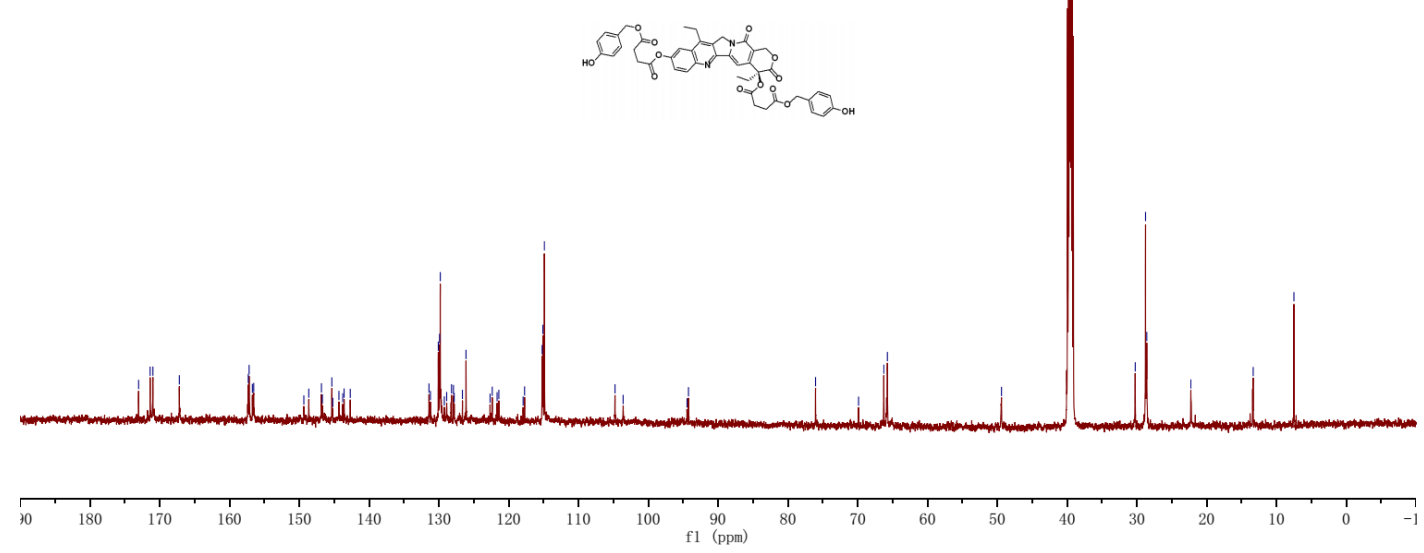

Figure S12. ${ }^{13} \mathrm{C}$ NMR spectrum of D1 (DMSO-d $)$. 


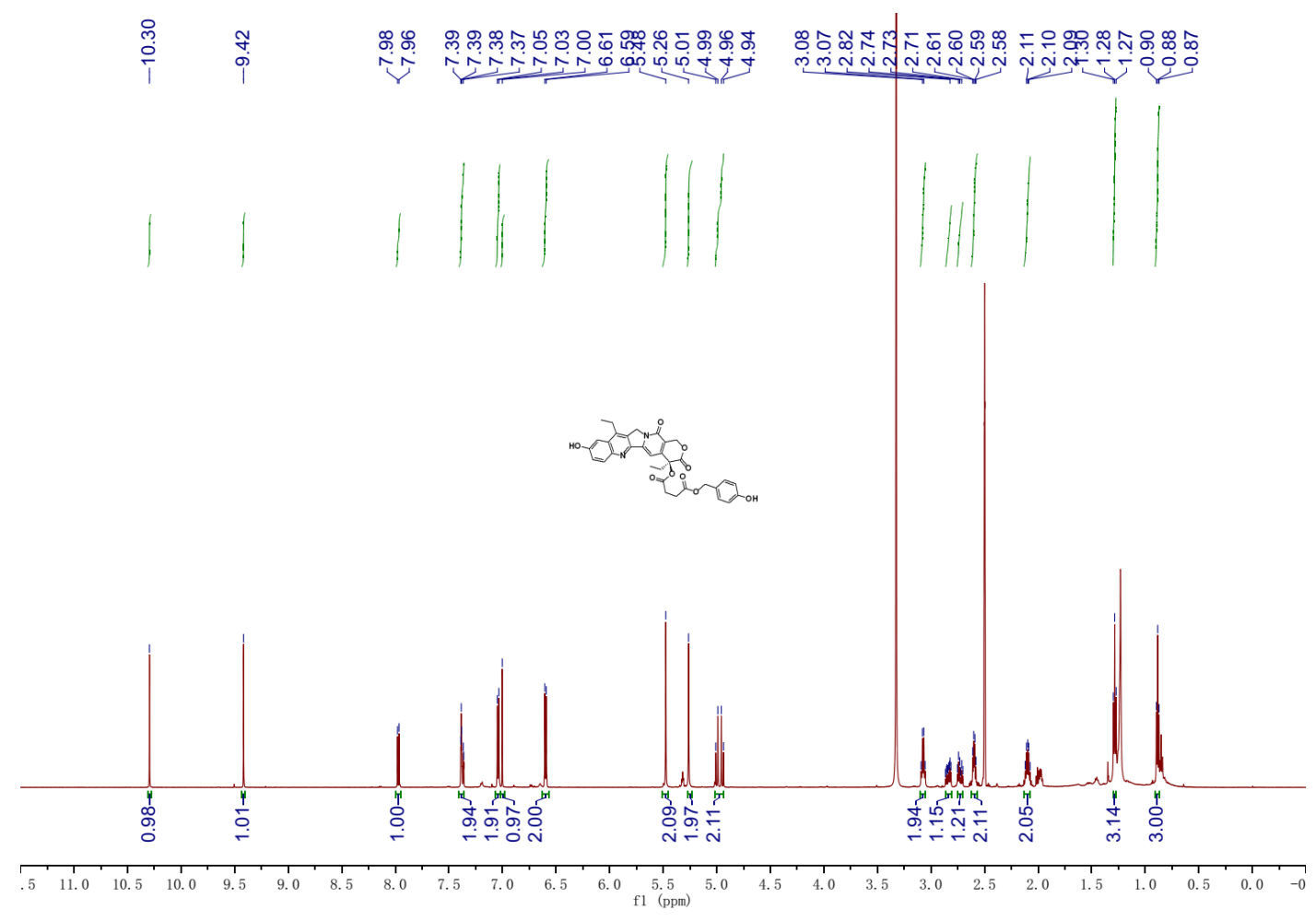

Figure S13. ${ }^{1}$ H NMR spectrum of D2 (DMSO-d6).

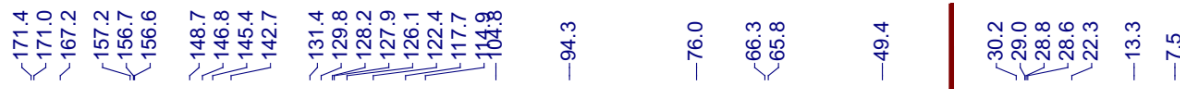
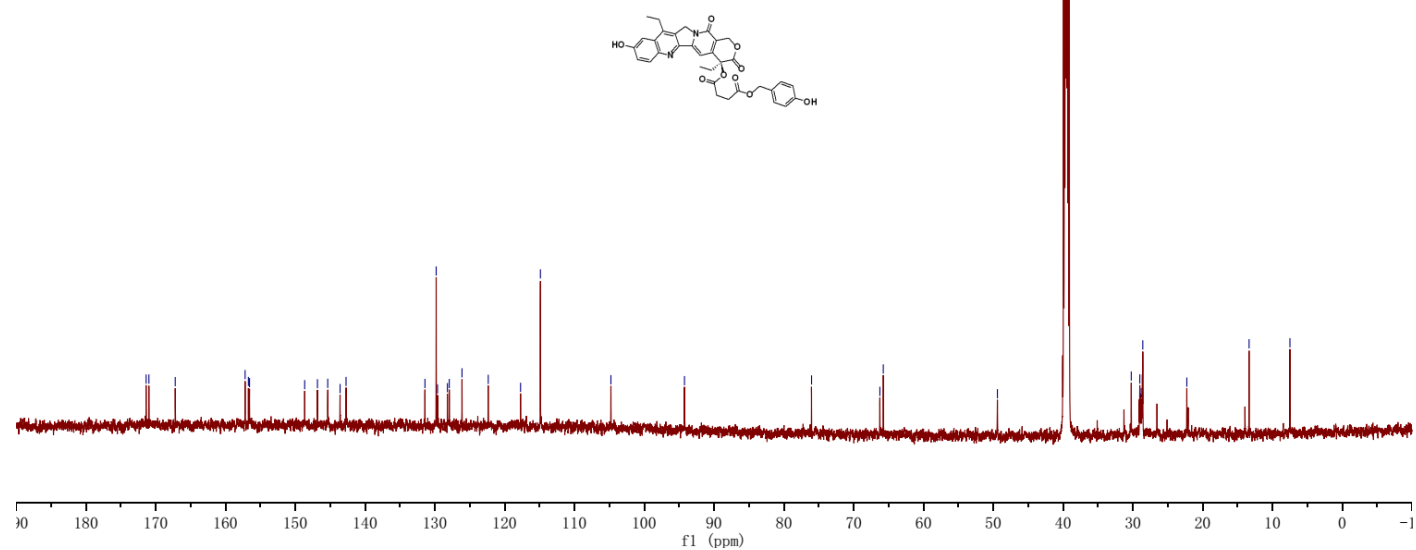

Figure S14. ${ }^{13} \mathrm{C}$ NMR spectrum of D2 (DMSO-d6). 
Item name: 3, Sample position: 1:F,3, Replicate number: 1

\begin{tabular}{|c|c|c|c|c|c|c|c|c|}
\hline . & Formula & $\begin{array}{l}\text { Neutral mass } \\
\text { (Da) }\end{array}$ & Observed m/z & $\begin{array}{l}\text { Mass error } \\
(\mathrm{mDa})\end{array}$ & $\begin{array}{l}\text { Mass error } \\
(\mathrm{ppm})\end{array}$ & Response & Adducts & Identification status \\
\hline 1 & c56h62b2n2o15 & 1024.43363 & 1025.44463 & 3.7 & 3.6 & 4071461 & $+\mathrm{H}_{1}+\mathrm{Na}$ & Identified \\
\hline 2 & c56h62b2n2o15 & 1024.43363 & 1025.44537 & 4.5 & 4.4 & 103674 & $+\mathrm{H},+\mathrm{Na}$ & Identified \\
\hline
\end{tabular}

Item name: 3

Channel name: Time $0.0901+/-0.0500$ minutes

Item description:

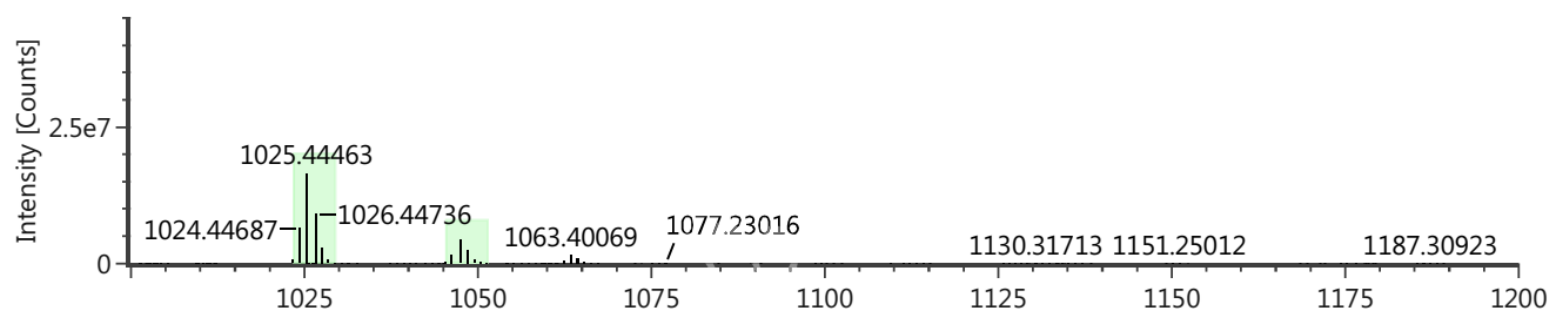

Item name: 3

Channel name: Time $0.9281+/-0.0500$ minutes

Figure S15. Electrospray Ionization high-resolution mass (ESI-MS) spectrum of SN38-SA-BP.

Item name: 4, Sample position: 1:F,4, Replicate number: 1

\begin{tabular}{|l|l|l|l|l|l|r|l|l|}
\hline. & Formula & $\begin{array}{l}\text { Neutral mass } \\
(\mathrm{Da})\end{array}$ & Observed $\mathrm{m} / \mathrm{z}$ & $\begin{array}{l}\text { Mass error } \\
(\mathrm{mDa})\end{array}$ & $\begin{array}{l}\text { Mass error } \\
(\mathrm{ppm})\end{array}$ & Response & Adducts & Identification status \\
\hline 1 & c44h42b2n2o15 & 860.27713 & 861.28658 & 2.2 & 2.5 & 2699061 & $+\mathrm{H}$ & Identified \\
\hline 2 & c44h42b2n2o15 & 860.27713 & 883.26766 & 1.3 & 1.5 & 584012 & $+\mathrm{Na}$ & Identified \\
\hline 3 & c44h42b2n2o15 & 860.27713 & 861.28667 & 2.3 & 2.6 & 242151 & $+\mathrm{H},+\mathrm{Na}$ & Identified \\
\hline
\end{tabular}

Item name: 4

Channel name: Time $0.1271+/-0.0500$ minutes

Item description:

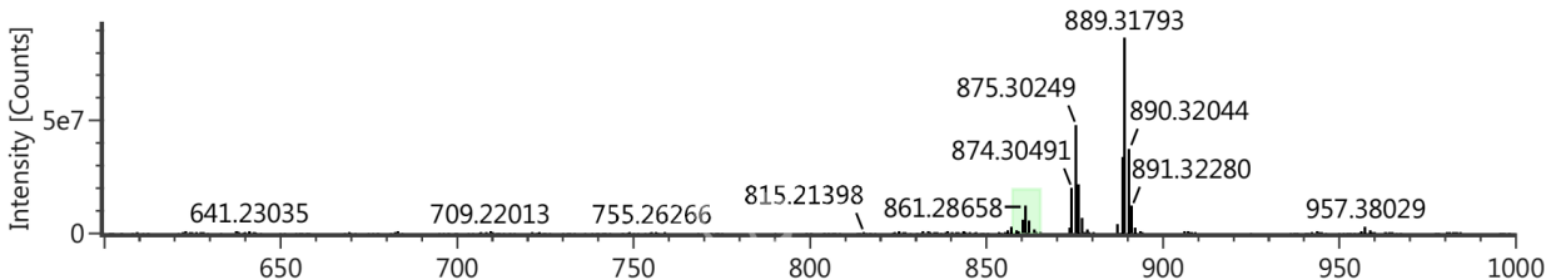

Observed mass $[\mathrm{m} / \mathrm{z}]$

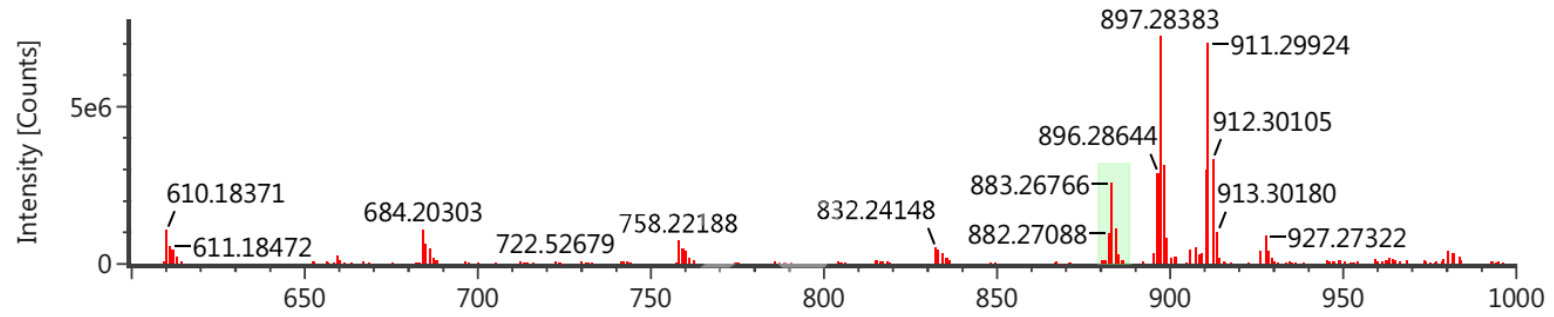

Figure S16. ESI-MS spectrum of SN38-SA-BA. 
Item name: $\mathrm{fx}-\mathrm{g}$, Sample position: 1:C,1, Replicate number: 1

\begin{tabular}{|l|l|l|l|l|l|c|l|l|}
\hline. & Formula & $\begin{array}{l}\text { Neutral mass } \\
(\mathrm{Da})\end{array}$ & Observed m/z & $\begin{array}{l}\text { Mass error } \\
(\mathrm{mDa})\end{array}$ & $\begin{array}{l}\text { Mass error } \\
(\mathrm{ppm})\end{array}$ & Response & Adducts & Identification status \\
\hline 1 & c44h40n2o13 & 804.25304 & 805.26240 & 2.1 & 2.6 & 1317339 & $+\mathrm{H},+\mathrm{Na}$ & Identified \\
\hline 2 & c44h40n2o13 & 804.25304 & 805.26249 & 2.2 & 2.7 & 887118 & $+\mathrm{H}$ & Identified \\
\hline 3 & c44h40n2o13 & 804.25304 & 805.26231 & 2.0 & 2.5 & 467159 & $+\mathrm{H},+\mathrm{Na}$ & Identified \\
\hline 4 & c44h40n2o13 & 804.25304 & 827.24452 & 2.3 & 2.7 & 162462 & $+\mathrm{Na}$ & Identified \\
\hline
\end{tabular}

Item name: $\mathrm{fx}-\mathrm{g}$

Channel name: Time $0.0831+/-0.0069$ minutes

Item description:

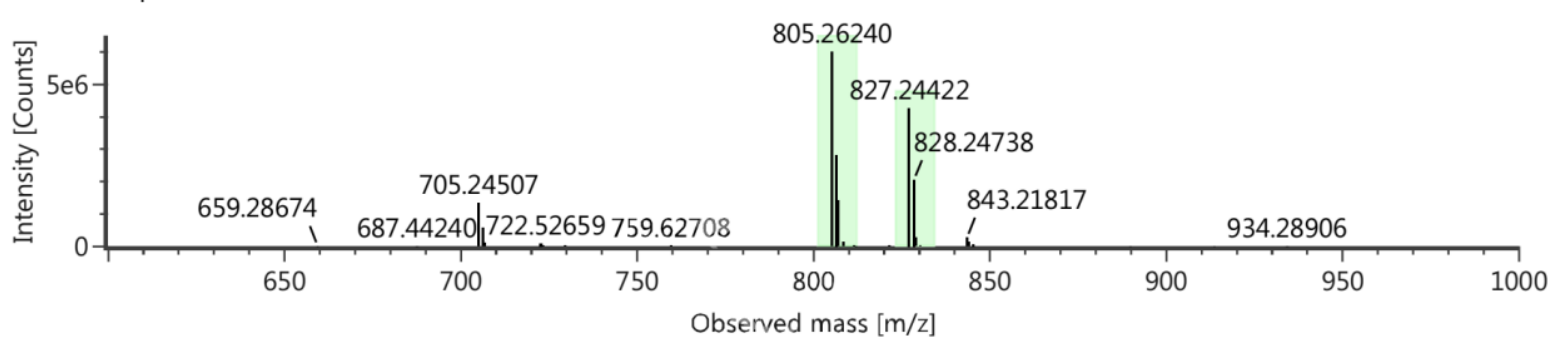

Figure S17. ESI-MS spectrum of D1.

Item name: $\mathrm{fx}-\mathrm{g}$, Sample position: 1:C,1, Replicate number: 1

\begin{tabular}{|l|l|l|l|l|l|r|l|l|}
\hline. & Formula & $\begin{array}{l}\text { Neutral mass } \\
(\mathrm{Da})\end{array}$ & Observed $\mathrm{m} / \mathrm{z}$ & $\begin{array}{l}\text { Mass error } \\
(\mathrm{mDa})\end{array}$ & $\begin{array}{l}\text { Mass error } \\
(\mathrm{ppm})\end{array}$ & Response & Adducts & Identification status \\
\hline 1 & c33h30n209 & 598.19513 & 599.20288 & 0.5 & 0.8 & 2524636 & $+\mathrm{H},+\mathrm{Na}$ \\
\hline 2 & c33h30n209 & 598.19513 & 599.20272 & 0.3 & 0.5 & 478928 & $+\mathrm{H},+\mathrm{Na}$ & Identified \\
\hline 3 & c33h30n209 & 598.19513 & 621.18454 & 0.2 & 0.3 & 306045 & $+\mathrm{Na}$ & Identified \\
\hline
\end{tabular}

Item name: $\mathrm{fx}-\mathrm{g}$

Channel name: Time $0.0981+/-0.0069$ minutes

Item description:
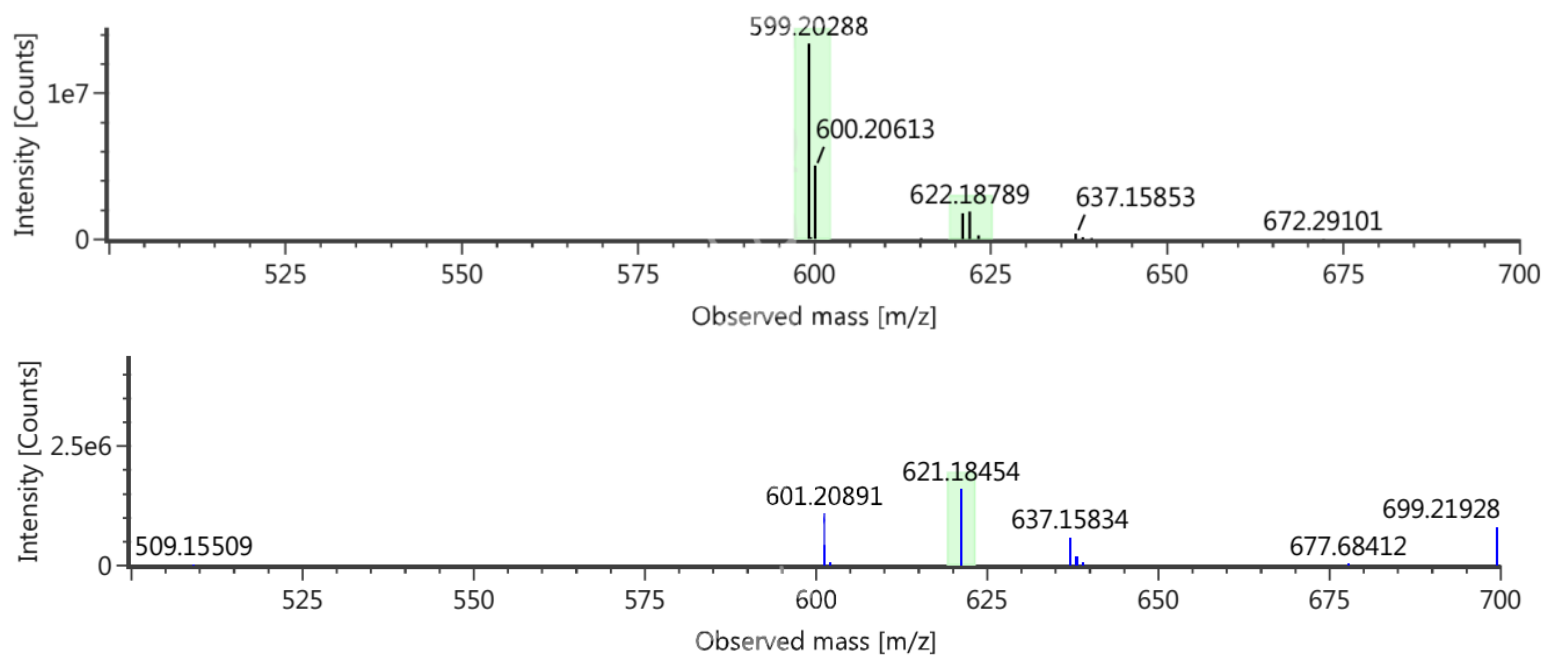

Figure S18. ESI-MS spectrum of D2. 